Reprod. Nutr. Dévelop., 1987, 27 (1 B), 227-228.

\title{
Vitesse de renouvellement du liquide du rumen mesurée par le polyéthylène glycol et le Co-EDTA à trois niveaux du tube digestif
}

M. DOREAU, Marie-Pierre LE GUEN, C. PONCET $\left(^{*}\right)$

Laboratoire de la Lactation

(*) Laboratoire de la Digestion des Ruminants, I.N.R.A. Theix, 63122 Ceyrat, France.

Summary. Rumen liquid turnover rate was estimated in cows from kinetics of concentration of PEG or Co-EDTA measured simultaneously at three sites of sampling : rumen, duodenum, feces. At each level, the two markers gave the same result, but turnover rate was greater when directly determined in rumen samples than indirectly in duodenal or fecal samples.

L'estimation de la vitesse de renouvellement $k$ ou du flux de liquide à la sortie du rumen peut être réalisée grâce à différents marqueurs solubles : PEG, $\mathrm{Cr}$ ou Co chélaté par I'EDTA, par mesure de l'évolution de leur concentration dans le rumen ou dans un compartiment postérieur. Nous avons comparé le PEG et le Co-EDTA chez la vache en déterminant $k$ à trois niveaux du tube digestif : rumen, duodénum et fèces.

Matériel et méthodes. Douze comparaisons ont été réalisées sur des vaches laitières munies de canules du rumen et du duodénum proximal. Six d'entre elles ont été réalisées sur 3 vaches (production laitière $(P L)$ moyenne : $15,9 \mathrm{~kg} / \mathrm{j}$ ) recevant un régime composé de foin $(40 \%)$ et de concentré $(60 \%)$ offert à volonté (matière sèche ingérée (MSI) moyenne : $15,8 \mathrm{~kg} / \mathrm{j}$ ) à $8 \mathrm{~h}$ et $20 \mathrm{~h}$ (régime $\mathrm{F}$ ); les 6 autres sur 3 autres vaches ( $P L$ moyenne $: 2,7 \mathrm{~kg} / \mathrm{j}$ ) recevant un régime composé d'ensilage de maïs $(60 \%)$ et de concentré $(40 \%)$ offert en quantités limitées (MSI moyenne : $8,0 \mathrm{~kg} / \mathrm{j}$ ) à $8 \mathrm{~h}$ et $16 \mathrm{~h}$, le concentré comprenant, pour 4 des mesures, $20 \%$ de suif (régime EM). Des doses uniques de PEG et de Co-EDTA ont été infusées dans le rumen à $8 \mathrm{~h}$. Leurs concentrations ont été déterminées respectivement par turbidimétrie et spectrophotométrie d'absorption atomique sur 10 à 13 échantillons de liquide du rumen (prélevés $1 \mathrm{~h} 30$ à $19 \mathrm{~h}$ après infusion), du duodénum (1 h 30 à $24 \mathrm{~h}$ après infusion) et de fèces (sur régime 1 seulement, $11 \mathrm{~h} 30$ à $72 \mathrm{~h}$ après infusion). Les estimations de $\mathrm{k}$ à partir des cinétiques ruminales, duodénales et fécales $(\mathrm{kr}, \mathrm{kd}, \mathrm{kf})$ ont été réalisées sur la phase de concentrations décroissantes selon un ajustement exponentiel et, pour kd, par ajustement au modèle à deux compartiments de Grovum et Williams (1973).

Résultats et discussion. Aucune différence significative n'a été mise en évidence entre les vitesses de renouvellement obtenues à partir des deux marqueurs (tabl. 1), ni aucune interaction entre le marqueur et le lieu de prélèvement (rumen ou duodénum) quel que soit le régime. Les deux marqueurs auraient ainsi les mêmes propriétés (éventuelle absorption ou fixation à la phase solide) dans les compartiments antérieurs au duodénum, du moins avec les régimes distribués. De légères différences non systématiques et dépendantes de la ration entre le PEG et le Cr-EDTA ou le Co-EDTA avaient été observées par Snyder et al. (1984) chez la vache et Poncet et al. (1986) chez le mouton. Les écarts-type résiduels de chaque estimation de $k$ ont été voisins pour les deux marqueurs. Les volumes d'eau du 
rumen estimés par le PEG et le Co-EDTA ont été non significativement différents $(73,7$ et $77,5 \mathrm{l})$, valeurs supérieures à celles obtenues par vidages complets du contenu du rumen, réalisé à $14 \mathrm{~h}$ quelques jours après les prélèvements $(65,5 \mathrm{l})$. A $14 \mathrm{~h}$, la concentration du marqueur a été toujours voisine de celle obtenue par l'ajustement. La précision de chaque estimation par marqueur a été comprise entre 0,6 et 3,8 I. La différence observée entre les méthodes rejoint les résultats de Snyder et al. (1984).

TABL. 1. - Estimation de la vitesse de renouvellement $k$ du liquide du rumen à trois niveaux du tube digestif.

\begin{tabular}{cccccc}
\hline $\begin{array}{c}\text { Lieu de } \\
\text { prélèvement }\end{array}$ & \multicolumn{2}{c}{ Rumen } & \multicolumn{2}{c}{ duodénum } & Fècès \\
\hline \multicolumn{1}{c}{ Régime } & $F$ & EM & F & EM & $F$ \\
\hline $\begin{array}{l}\text { k PEG }\left(h^{-1}\right) \\
\begin{array}{l}\text { k Co-EDTA } \\
\left(h^{-1}\right)\end{array}\end{array}$ & $0,120 \pm 0,002$ & $0,089 \pm 0,022$ & $0,105 \pm 0,008$ & $0,079 \pm 0,021$ & $0,108 \pm 0,010$ \\
\hline
\end{tabular}

$\mathrm{kr}$ a été significativement plus élevée que $k d(P<0,01)$. L'équation reliant les estimations réalisées aux deux niveaux s'écrit $k d=0,815 \mathrm{kr}+0,007$ $(r=0,90)$. II $n^{\prime} a$ pas été possible de relier cette différence à une caractéristique de la ration. Aucune différence n'a été observée entre kd et $\mathrm{kf}$. Ces résultats confirment ceux de Robinson et Sniffen (1983) obtenus sur vaches. En revanche, sur taurillons ou moutons, aucune différence n'avait été mise en évidence par Faichney et Griffiths (1978) et Poncet et al. (1986) entre kr et kd, et par Teeter et al. (1981) et Mira et MacRae (1982) entre kr et kf. II est possible que ces variations entre espèces soient dues à des différences dans l'absorption de marqueur ou dans les échanges d'eau au niveau d'un compartiment de mélange. Le choix du modèle expérimental pourrait également les expliquer (Snyder et al., 1984). La précision de la détermination de $\mathrm{k}$ (écart-type résiduel) a été la même aux trois lieux de prélèvement.

L'utilisation du modèle de Grovum et Williams (1973) pour estimer kd a donné des résultats non significativement différents de l'ajustement exponentiel. Cependant, pour 3 comparaisons sur le régime EM (valeurs de $k$ faibles), un plateau de concentration au niveau duodénal a été observé entre 3 et $9 \mathrm{~h}$ après l'infusion ; l'emploi d'un modèle à deux compartiments semble alors inadéquat. Les estimations de la vitesse de renouvellement au niveau du compartiment de mélange secondaire (caillette) sont de 1,1 $\pm 0,4$ et $0,7 \pm 0,2 \mathrm{~h}^{-1}$ pour les régimes F et EM, et celles du temps de délai entre la sortie du rumen et l'entrée dans le duodénum (feuillet) de $0,8 \pm 0,3$ et $0,6 \pm 0,1 \mathrm{~h}$. Ces valeurs, bien qu'obtenues indirectement, concordent avec différentes estimations de la bibliographie.

En conclusion, les deux marqueurs de la phase liquide étudiés ont eu le même comportement dans le tractus digestif, mais l'estimation de la vitesse de renouvellement de l'eau du rumen a varié en fonction du lieu de prélèvement, indépendamment du régime alimentaire des vaches.

Faichney G. J., Griffiths D. A., 1978. Br. J. Nutr., 40, 71-82.

Grovum W. L., Williams V. J., 1973. Br. J. Nutr., 30, 313-329.

Mira J. J. F., MacRae J. C., 1982. Proc. Nutr. Soc., 41, 77A.

Poncet C., Beaufort M. T., Al Abd A., 1986. Reprod. Nutr. Dévelop., 26, 321-322.

Robinson P. H., Sniffen C. J., 1983. J. Dairy Sci., 66, suppl. 1, 187-188.

Snyder T. J., Muller L. D., Rogers J. A., Abrams S. M., 1984. J. Dairy Sci., 67, 1953-1964.

Teeter R. G., Owens F. N., Sharp M. W., 1981. J. anim. Sci., 53, suppl. 1, 437 